\title{
BMJ Open Trends of CT utilisation in an emergency department in Taiwan: a 5-year retrospective study
}

\author{
Sung-Yuan $\mathrm{Hu},{ }^{1,2,3,4,5,6,7}$ Ming-Shun Hsieh, ${ }^{5,8,9,10}$ Meng-Yu Lin, ${ }^{11}$ \\ Chiann-Yi Hsu, ${ }^{12}$ Tzu-Chieh Lin, ${ }^{1}$ Chorng-Kuang How, ${ }^{5,9}$ Chen-Yu Wang, ${ }^{13}$ \\ Jeffrey Che-Hung Tsai, ${ }^{1,5}$ Yu-Hui Wu, ${ }^{14,15,16}$ Yan-Zin Chang ${ }^{3,4,17}$
}

To cite: Hu S-Y, Hsieh M-S, Lin M-Y, et al. Trends of CT utilisation in an emergency department in Taiwan: a 5-year retrospective study. BMJ Open 2016;6:e010973. doi:10.1136/bmjopen-2015010973

- Prepublication history for this paper is available online. To view these files please visit the journal online (http://dx.doi.org/10.1136/ bmjopen-2015-010973).

Received 24 December 2015 Revised 23 March 2016 Accepted 24 March 2016

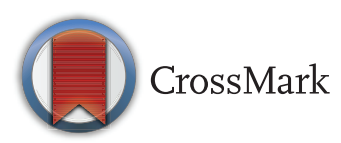

For numbered affiliations see end of article.

Correspondence to Professor Yan-Zin Chang; yzc@csmu.edu.tw

\section{ABSTRACT}

Objectives: To investigate the association between the trends of CT utilisation in an emergency department (ED) and changes in clinical imaging practice and patients' disposition.

Setting: A hospital-based retrospective observational study of a public 1520-bed referral medical centre in Taiwan.

Participants: Adult ED visits (aged $\geq 18$ years) during 2009-2013, with or without receiving CT, were enrolled as the study participants.

Main outcome measures: For all enrolled ED visits, we retrospectively analysed: (1) demographic characteristics, (2) triage categories, (3) whether CT was performed and the type of CT scan, (4) further ED disposition, (5) ED cost and (6) ED length of stay.

Results: In all, 269239 adult ED visits (148 613 male patients and 120626 female patients) were collected during the 5-year study period, comprising $38609 \mathrm{CT}$ scans. CT utilisation increased from $11.10 \%$ in 2009 to $17.70 \%$ in 2013 (trend test, $p<0.001$ ). Four in 5 types of CT scan (head, chest, abdomen and miscellaneous) were increasingly utilised during the study period. Also, CT was increasingly ordered annually in all age groups. Although ED CT utilisation rates increased markedly, the annual ED visits did not actually increase. Moreover, the subsequent admission rate, after receiving ED CT, declined (59.9\% in 2009 to $48.2 \%$ in 2013).

Conclusions: ED CT utilisation rates increased significantly during 2009-2013. Emergency physicians may be using $\mathrm{CT}$ for non-emergent studies in the ED. Further investigation is needed to determine whether increasing CT utilisation is efficient and cost-effective.

\section{INTRODUCTION}

Making a prompt and correct diagnosis in an overloaded emergency department (ED) is a great challenge for emergency physicians. CT is one of the most widely used imaging technologies in the ED because of its superior diagnostic ability and widespread accessibility. The CT scan can provide clear imaging

\section{Strengths and limitations of this study}

- We used a large database with detailed information from a medical centre in Taiwan to describe the current trends of emergency department (ED) CT utilisation.

- The data are valuable in clinical practice since the institute analysed in the current study is one of the most crowded EDs in Taiwan. The results showed an association between the trends of $E D$ CT utilisation and the disposition of ED patients. The use of multiple CT scans during an ED visit was also investigated.

- The limitations of this study were the retrospective design and confounding factors that could not be excluded.

- Cultural concerns, although important for fully understanding this study, could not be completely quantified via statistical analysis.

- As the most ED visits were made by older patients $(48.03 \%)$, who tended to have multiple problems, classification of ED visits according to their symptoms was difficult.

of the pathological lesion in a patient and sometimes an incidental finding during a CT scan may allow earlier treatment of a disease. However, frequent CT utilisation has raised concerns about its necessity because of the cost, radiation exposure, potential allergy reaction and risk of contrast-induced nephropathy $(\mathrm{CIN}) .^{1-9}$

Several large studies, mainly from Western countries, have described a steady increase in CT utilisation in the ED. ${ }^{1-9}$ Two reports from Asian countries also described similar trends of CT utilisation in the ED. ${ }^{10}{ }^{11}$ The rate of CT utilisation in the ED ranged from $2.8 \%$ to $13.9 \%$ in the USA during $1995-2007 ; 12.4 \%$ to $33 \%$ in South Korea during 2001-2010; and $9.8 \%$ to $13.9 \%$ in China during 20052008. These data indicate that the global trend of ED CT utilisation is increasing. 
The major difference of ED CT utilisation in Taiwan compared with that in Western countries is that ED physicians in Taiwan treat referral patients from other hospitals or patients with emergent conditions, they as well as treat ambulatory patients with non-emergent problems such as acute upper respiratory infection or acute gastroenteritis. ${ }^{12} 13$ Also, the National Health Insurance system in Taiwan covers the fee for basic laboratory investigations, radiological survey and clinical management of an ED course, but does not include the registration fee (an administrative charge payable at each visit amounting to about US\$23 in the EDs of most medical centres). Thus, EDs in Taiwan are frequently crowded and the model of medical practice tends to differ from that in Western countries, including the utilisation of ED CT. ${ }^{1415}$

Furthermore, the ED length of stay (LOS) in Taiwan is longer than in most Western countries. The possible factors of prolonged ED LOS include the time waiting in the ED for the final reports and subsequent consultation at the request of patients or their family after the CT scan, especially in patients with minor or non-critical findings whose hospital admission was not necessary. As they must receive further observation in the ED, the ED LOS is prolonged. ${ }^{10}$

The aim of this study was to investigate the association between the trends of ED CT utilisation and changes in clinical imaging practice and patients' disposition in a medical centre in Taiwan.

\section{METHODS}

This retrospective observational study was conducted in a public 1520-bed referral medical centre in Taiwan. The data for the adult ED visits (aged $\geq 18$ years) during 2009-2013 were retrieved from the hospital's electronic database. The ED visits with incomplete data were excluded from the study. In this study, we analysed the data on the basis of per visit rather than per patient.

As patients' identifying data were scrambled to ensure privacy before being released to researchers, this study was exempted from obtaining informed consent from participants.

All ED visits were classified using the Taiwan Triage Acuity Scale, which included the following five categories of severity: resuscitation, emergent, urgent, less urgent and not urgent. CT scan in our ED was available throughout the day. Orders for CT scan in the ED were at the sole clinical discretion of the physicians.

The types of CT scans were classified according to the body region, such as head, chest, abdomen, spine and miscellaneous. The spinal CTs included cervical-, thoracicand lumbar-sacral scans. CT angiographies of the pulmonary artery and aorta were included in the category of chest CT. Miscellaneous CTs included various uncommon CTs, such as single extremity and thyroid scans. When an ED patient received more than one CT scan during the ED stay, the visit was classified as one in which multiple CT scans were given. All CTs were divided into contrast-enhanced and non-contrast-enhanced scans. During the study periods, all the patients received nonionic contrast medium for CT image enhancement. The average cost of a CT was US $\$ 169.52$ per body region without contrast, and US\$213.99 per body region with contrast.

The definition of subsequent ward admission was an admission of an ED patient to the general ward or intensive care unit (ICU) via ED. The ED LOS was defined as an ED course from registration in the ED to discharge or subsequent admission. The ED LOS and cost were recorded for all visits. If the time of ED LOS was less than the median ED LOS, it was classified as a short ED LOS. If the total fee of an ED course was more than the median ED cost, it was classified as low ED cost.

The primary outcome of this study was the annual trend of ED CT utilisation for adult ED visits during 2009-2013. The following data were analysed for each ED visit: (1) demographic characteristics, (2) triage category, (3) whether CT was performed and the type of CT scan, (4) frequency of CT scan during a visit, (5) disposition of ED visits, (6) cost and (7) ED LOS.

For comparison, the levels of triage acuity were dichotomised into high (resuscitation, emergent and urgent) and low (less urgent and not urgent) acuity. The median values of the ED cost and LOS were used as the cut-off values to transform the data into categorical variables (high/low ED cost and long/short ED LOS, respectively) for analyses.

The comparisons of the categorical data between ED visits with or without CT utilisation were conducted using $\chi^{2}$ tests. Annual difference for ED CT utilisation was examined using trend tests. Significance was defined as a $\mathrm{p}$ value of $<0.05$. All the statistical analyses were performed using the SAS 9.4 statistical package (SAS Institute Inc, Cary, North Carolina, USA).

\section{RESULTS}

After $288 \mathrm{ED}$ visits were excluded due to incomplete data, a total of 269239 ED visits (148 613 male visits and 120626 female visits; median age 55.6 years) during the 5 -year period were analysed, and these visits resulted in 38609 CT scans. Our ED visits were divided into the following four categories: internal medicine, trauma, obstetrics and paediatrics. Throughout the study period, internal medicine accounted for $73.7 \%$ of CT scans, trauma $22.7 \%$ and obstetrics $3.6 \%$. The median ED LOS and cost were 17.8 hours and US $\$ 130.52$ per visit, respectively. ED courses shorter than 17.8 hours were classified as short ED LOS. If the total fee of an ED course was $>$ US $\$ 130.52$, it was classified as low cost. The annual distributions of age, gender, CT utilisation, triage category, cost, ED LOS and disposition of ED visits are summarised in table 1.

We further calculated the waiting time for ED CT and found a median duration of 1.62 hours from registration in the ED to commencement of CT scan. However, the 
Table 1 Demographic characteristics, CT utilisation, triage category, cost, LOS and disposition of emergency department visits during 2009-2013

\begin{tabular}{|c|c|c|c|c|c|c|c|}
\hline & $\begin{array}{l}2009-2013 \\
(n=269239) \\
n(\%)\end{array}$ & $\begin{array}{l}2009 \\
(n=51381) \\
n(\%)\end{array}$ & $\begin{array}{l}2010 \\
(n=53908) \\
n(\%)\end{array}$ & $\begin{array}{l}2011 \\
(n=54832) \\
n(\%)\end{array}$ & $\begin{array}{l}2012 \\
(n=55670) \\
n(\%)\end{array}$ & $\begin{array}{l}2013 \\
(n=53448) \\
n(\%)\end{array}$ & p Value \\
\hline CT utilisation & & & & & & & $<0.001^{*}$ \\
\hline No & $230630(85.7)$ & 45670 (88.9) & 46817 (86.9) & 46928 (85.6) & 47234 (84.9) & 43981 (82.3) & \\
\hline Yes & 38609 (14.3) & $5711(11.1)$ & 7091 (13.2) & 7904 (14.4) & 8436 (15.2) & 9467 (17.7) & \\
\hline Gender & & & & & & & $<0.001^{*}$ \\
\hline Male & $148613(55.2)$ & 28898 (56.2) & $30133(55.9)$ & $30431(55.5)$ & 30211 (54.3) & $28940(54.2)$ & \\
\hline Female & $120626(44.8)$ & 22483 (43.8) & $23775(44.1)$ & 24401 (44.5) & 25459 (45.7) & $24508(45.9)$ & \\
\hline Age (years) & & & & & & & $<0.001^{*}$ \\
\hline$\geq 18$ to $<40$ & 78967 (29.3) & 14795 (28.8) & $15636(29.0)$ & 16399 (29.9) & 16599 (29.8) & $15538(29.1)$ & \\
\hline$\geq 40$ to $<65$ & 93316 (34.7) & 17401 (33.9) & 18410 & 18978 (34.6) & $19514(35.1)$ & 19013 (35.6) & \\
\hline$\geq 65$ & $96956(36.0)$ & 19185 (37.3) & $19862(36.8)$ & 19455 (35.5) & $19557(35.1)$ & $18897(35.4)$ & \\
\hline Triage category & & & & & & & $<0.001^{*}$ \\
\hline High & 248413 (92.3) & 51224 (99.7) & 51984 (96.4) & $49380(90.1)$ & 51035 (91.7) & 44790 (83.8) & \\
\hline Low & $20826(7.7)$ & $157(0.3)$ & 1924 (3.6) & $5452(9.9)$ & 4635 (8.3) & 8658 (16.2) & \\
\hline Disposition & & & & & & & $<0.001^{*}$ \\
\hline Admitted & 68216 (25.3) & $13158(25.6)$ & $14318(26.6)$ & 13755 (25.1) & $13908(25.0)$ & 13077 (24.5) & \\
\hline Discharged & $187632(69.7)$ & 36332 (70.7) & $37284(69.2)$ & 38038 (69.4) & $38908(69.9)$ & $37070(69.4)$ & \\
\hline Transferred & $690(0.3)$ & $140(0.3)$ & $112(0.2)$ & $112(0.2)$ & $125(0.2)$ & $201(0.4)$ & \\
\hline DAMA & $11350(4.2)$ & $1532(3.0)$ & 1905 (3.5) & $2620(4.8)$ & $2470(4.4)$ & 2823 (5.3) & \\
\hline Expired & $1351(0.5)$ & $219(0.4)$ & $289(0.5)$ & $307(0.6)$ & $259(0.5)$ & $277(0.5)$ & \\
\hline LOS & & & & & & & $<0.001^{*}$ \\
\hline Short & $198372(73.7)$ & 39689 (77.2) & $39886(74.0)$ & $39678(72.4)$ & $40842(73.4)$ & $38277(71.6)$ & \\
\hline Long & 70867 (26.3) & 11692 (22.8) & $14022(26.0)$ & $15154(27.6)$ & $14828(26.6)$ & 15171 (28.4) & \\
\hline Cost & & & & & & & $<0.001^{*}$ \\
\hline Low & $142110(52.8)$ & $28823(56.1)$ & $29115(54.0)$ & $27556(50.3)$ & $27110(48.7)$ & $29506(55.2)$ & \\
\hline High & $127129(47.2)$ & 22558 (43.9) & $24793(46.0)$ & $27276(49.7)$ & $28560(51.3)$ & $23942(44.8)$ & \\
\hline
\end{tabular}

median ED LOS for visits with ED CT utilisation was 19.64 hours, which was significantly longer than for those without ED CT utilisation $(4.59$ hours $)(p<0.001)$. The median cost of ED visits with CT utilisation was US \$272.27, which was higher than those without CT utilisation $(p<0.001)$. Further comparisons of age, gender, triage category, cost, ED LOS and dispositions of ED visits, with and without CTs, are summarised in table 2. Significant differences between the two groups were found for all variables $(p<0.001)$.

Types of CT, use of contrast medium and frequency of CT scan during an ED visit are summarised in figure 1 and table 3. In total, in $95.91 \%$ of ED visits, a single CT scan was given, whereas in $4.09 \%$ of the visits, multiple CTs were given. The frequency of multiple CT scans during an ED visit also steadily increased from $4.09 \%$ in 2009 to $5.05 \%$ in 2013 (trend test, $\mathrm{p}<0.001$ ). With respect to body regions, head $(44.74 \%)$ and abdomen (37.94\%) accounted for the greatest proportion of CT utilisation, followed by chest $(10.01 \%)$, miscellaneous $(5.86 \%)$ and spine $(1.45 \%)$. Contrast-enhanced CTs were ordered in more than half of the ED visits $(63.21 \%)$. There was a significant annual increase in the utilisation of head, abdomen, chest and miscellaneous CT scans in the ED in our study (trend test, $\mathrm{p}<0.001$ ) (figure 1). However, spine CT scans remained at a low annual rate of utilisation.

Among the three age groups, the greatest number of CT scans ordered by ED physicians was for patients aged $\geq 65$ years. All age groups showed a steady annual increase in ED CT utilisation rates (trend test, $\mathrm{p}<0.001$ ) (figure 2).

The relationship between the trends of annual numbers of ED visits and CT utilisation are delineated in figure 3. The annual ED visits during the study period were 51381 in 2009, 53908 in 2010, 54832 in 2011, 55670 in 2012 and 53448 in 2013. The average rate of ED CT utilisation in the study period was $14.3 \%$; the rate was $11.1 \%$ in 2009 , $13.2 \%$ in $2010,14.4 \%$ in $2011,15.2 \%$ in 2012 and $17.7 \%$ in 2013, respectively. The increase in ED CT utilisation was significantly greater than the increase in ED visits.

The annual trend of association between ED CT utilisation and subsequent admission rate is delineated in figure 3. In all, $68216 \mathrm{ED}$ patients were subsequently admitted, including those admitted to the ICU. Although the overall rate of ED CT utilisation had markedly increased during the 5-year period (11.1\% in 2009 to $17.7 \%$ in 2013), the subsequent admission rate among ED visits with CT utilisation did not increase accordingly, but, rather, declined from $59.9 \%$ in 2009 to $48.2 \%$ in 
Table 2 Comparison between emergency department visits with and without $C T$ utilisation

\begin{tabular}{|c|c|c|c|}
\hline & $\begin{array}{l}\text { Without CT } \\
\text { utilisation } \\
(n=230630) \\
n(\%)\end{array}$ & $\begin{array}{l}\text { With CT } \\
\text { utilisation } \\
(n=38609) \\
n(\%)\end{array}$ & p Value \\
\hline Age (years) & & & $<0.001^{*}$ \\
\hline$\geq 18$ to $<40$ & 72888 (31.60) & 6079 (15.75) & \\
\hline$\geq 40$ to $<65$ & 79328 (34.40) & 13988 (36.23) & \\
\hline$\geq 65$ & $78414(34.00)$ & $18542(48.03)$ & \\
\hline Gender & & & $<0.001^{*}$ \\
\hline Male & 125299 (54.33) & $23314(60.38)$ & \\
\hline Female & 105331 (45.67) & 15295 (39.62) & \\
\hline Disposition & & & $<0.001^{*}$ \\
\hline Admitted & $\begin{array}{r}47575(20.63) \\
172252(74.69)\end{array}$ & $\begin{array}{l}20641(53.46) \\
15380(39.84)\end{array}$ & \\
\hline Discharged & $503(0.22)$ & $187(0.48)$ & \\
\hline $\begin{array}{l}\text { Transferred } \\
\text { DAMA } \\
\text { Expired }\end{array}$ & $\begin{array}{l}9187(3.98) \\
1113(0.48)\end{array}$ & $\begin{array}{r}2163(5.60) \\
238(0.62)\end{array}$ & \\
\hline $\begin{array}{l}\text { Triage categor } \\
\text { High } \\
\text { Low }\end{array}$ & $\begin{array}{l}210980(91.48) \\
19650(8.52)\end{array}$ & $\begin{array}{c}37433(96.95) \\
1176(3.05)\end{array}$ & $<0.001^{*}$ \\
\hline $\begin{array}{l}\text { LOS } \\
\text { Short } \\
\text { Long }\end{array}$ & $\begin{array}{r}180145(78.11) \\
50485(21.89)\end{array}$ & $\begin{array}{l}18227(47.21) \\
20382(52.79)\end{array}$ & $<0.001^{*}$ \\
\hline $\begin{array}{l}\text { Cost } \\
\text { Low } \\
\text { High }\end{array}$ & $\begin{array}{r}136981(59.39) \\
93649(40.61)\end{array}$ & $\begin{array}{r}5129(13.28) \\
33480(86.72)\end{array}$ & $<0.001^{*}$ \\
\hline
\end{tabular}

2013. Pearson's correlation analysis was conducted to examine the relationship between the annual ED CT utilisation rate and the subsequent admission rate after ED CT utilisation, and it showed a strongly negative correlation between the two trends $(\mathrm{r}=-0.778, \mathrm{p}<0.001)$.

\section{DISCUSSION}

The results of our study demonstrate an increasing rate of ED CT utilisation (11.0-17.70\% during 2009-2013) in adult ED visits. An increasing trend of ED CT utilisation was observed for head, chest, abdomen and miscellaneous scans. The trend was also observed in all age groups. However, annual numbers of ED visits did not actually increase. Moreover, the subsequent admission rate declined for ED visits with CT scans.

Head CT was the major type of CT utilisation in our ED, which was similar to the results reported in other studies. ${ }^{11} 16$ Interestingly, a large proportion of the head CTs were ordered for ED visits with non-specific symptoms, and even for patients with normal neurological physical examinations. ${ }^{17-20}$ Abdominal CT was the second most common type of CT utilisation, mostly for ED visits with complicated abdominal pain or fever of unknown origin. Chest CT also showed an increasing rate of utilisation. These findings imply that ED physicians in Taiwan frequently order chest CTs for the survey of ambiguous chest pain, possibly owing to fear of lawsuits due to misdiagnosis or delayed diagnosis of aortic dissection. All four types of CT were utilised increasingly year by year. In our opinion, non-clinical factors may be influencing physicians' decision-making for CT utilisation. Therefore, CTs were sometimes ordered at the ED physicians' discretion with a view to confirming the clinical condition of the patients, even in cases where there was only minimal uncertainty. A number of factors may explain the number of orders for ED CT scans. It is possible that younger physicians, due to lack of clinical experience, order CT imaging more frequently. Likewise, ED visits during peak hours might tend to have a higher possibility of resulting in a CT scan because prompt screening could expedite the throughput of the ED visit. ${ }^{10}$

Although cervical spine CT has become a popular diagnostic tool for trauma patients with suspected
Figure 1 OR for CT frequency based on CT type (2009 as reference).

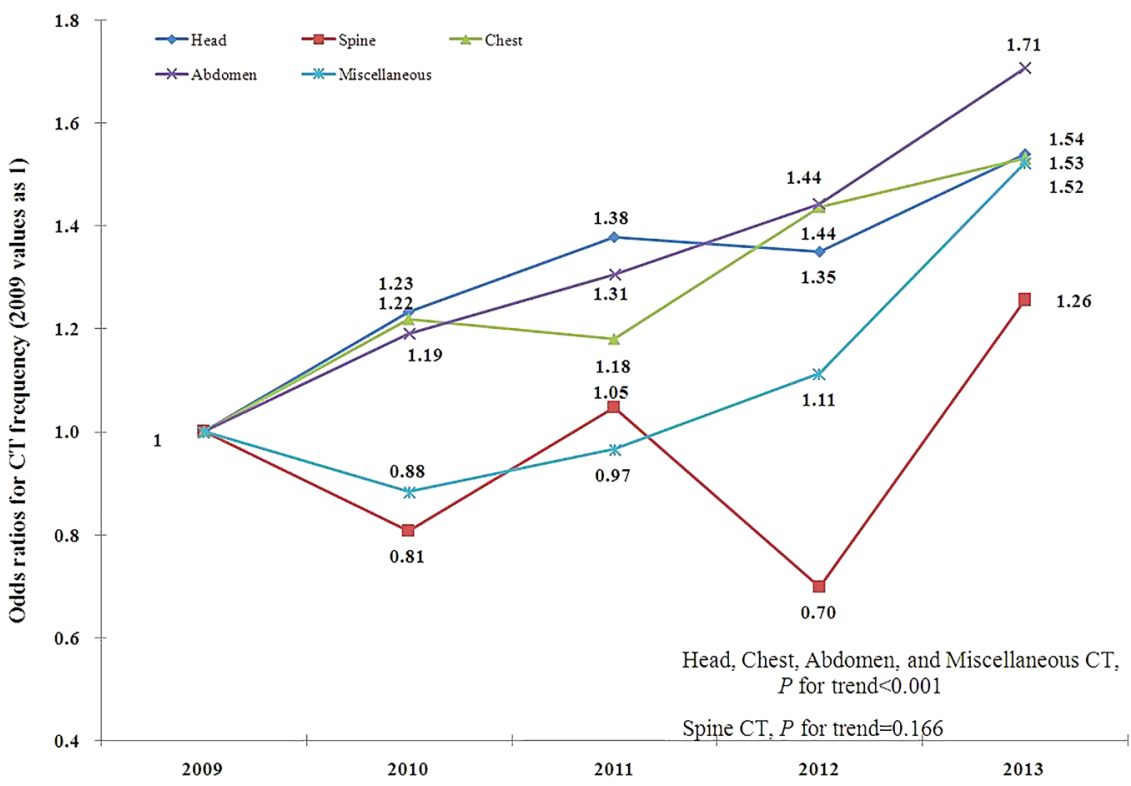




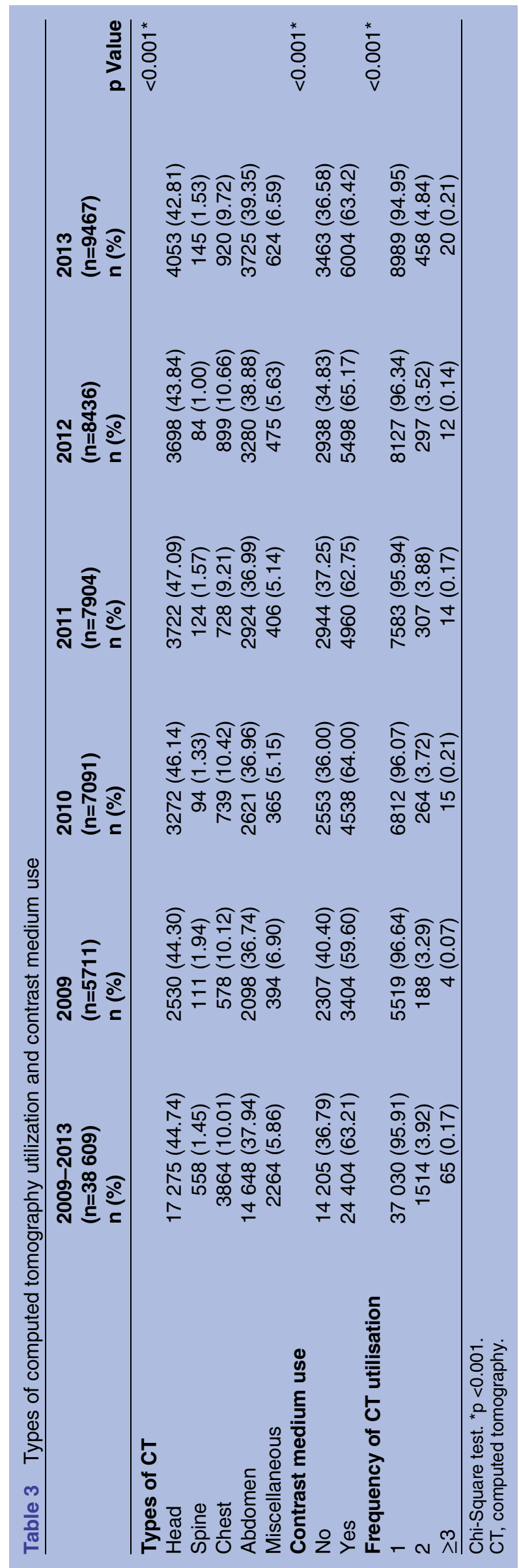

cervical spine injuries, ED physicians more frequently ordered MRI to survey associated injuries in our hospital, due to its accessibility. ${ }^{21} 22$

Other factors may also account for the increasing rate of ED CT utilisation, as follows: (1) if a patient had to stay in a crowded ED for prolonged observation, the physicians might order a CT in order to make a rapid diagnosis, thereby facilitating the patient's disposition; (2) a prolonged time waiting for admission resulted in the CT scan being performed in the ED rather than in the ward. As one of the most crowded EDs in Taiwan during the study period, $9.3 \%$ of our ED visits by patients who needed admission had a wait time of $>48$ hours. The incremental rate of ED CT utilisation could be explained by difficulty admitting patients, rather than the emergent necessity of an imaging study.

The Pearson's correlation analysis in figure 3 shows a strongly negative correlation between the two trends, namely, of ED CT utilisation rate and subsequent admission rate. There exist two basic scenarios: (1) CT overuse and (2) avoidance of admission due to CT use in the ED. We assumed that the patients were ill enough to undergo evaluation by CT scan; however, a large number of these patients were discharged later. This might mean that CT scans were increasingly used as screening tools. ED CT reduced the necessity of admission in certain situations. For example, some patients with head injury who did not require admission for observation of a neurological condition would benefit from a CT scan. ED acuity steadily increased during the study period, but the rate of hospital admission (25.6\% in $2009,26.6 \%$ in $2010,25.1 \%$ in $2011,25.0 \%$ in 2012 and $24.5 \%$ in 2013) remained stable (table 1). However, the annual utilisation of ED CT scans increased from $11.1 \%$ to $17.7 \%$ (figure 3 ), so CT overuse might be the more likely scenario. Inappropriate referrals or patients' requests for CT scans could explain the trends, and there was a large proportion of $\mathrm{ED}$ visits by patients who received a CT scan for rapid screening. It is not our intention here to apportion blame for the utilisation of ED CT scans, but we believe it is important that the issue of unnecessary CT scans in the ED be addressed.

In Taiwan's EDs, patients or their families often request advanced imaging examinations through the National Health Insurance system, even when such a study was not suggested by the treating doctor. Owing to cultural factors and to avoid potential lawsuits, ED physicians often agree with these requests. Also, many of our ED visits were referred from other hospitals, for further management, or for a second opinion. Although the CT images were taken in other hospitals, they could be shared. In 2010, Taiwan's Ministry of Health and Welfare announced that CT scans repeated within 90 days, including those performed in another hospital, would not be covered by the National Health Insurance system. Repeat CT scans were still frequently conducted in our ED. Possible explanations include: (1) a gap existing in CT scan quality between different facilities; (2) 
Figure 2 OR for CT frequency based on age group (2009 as reference).

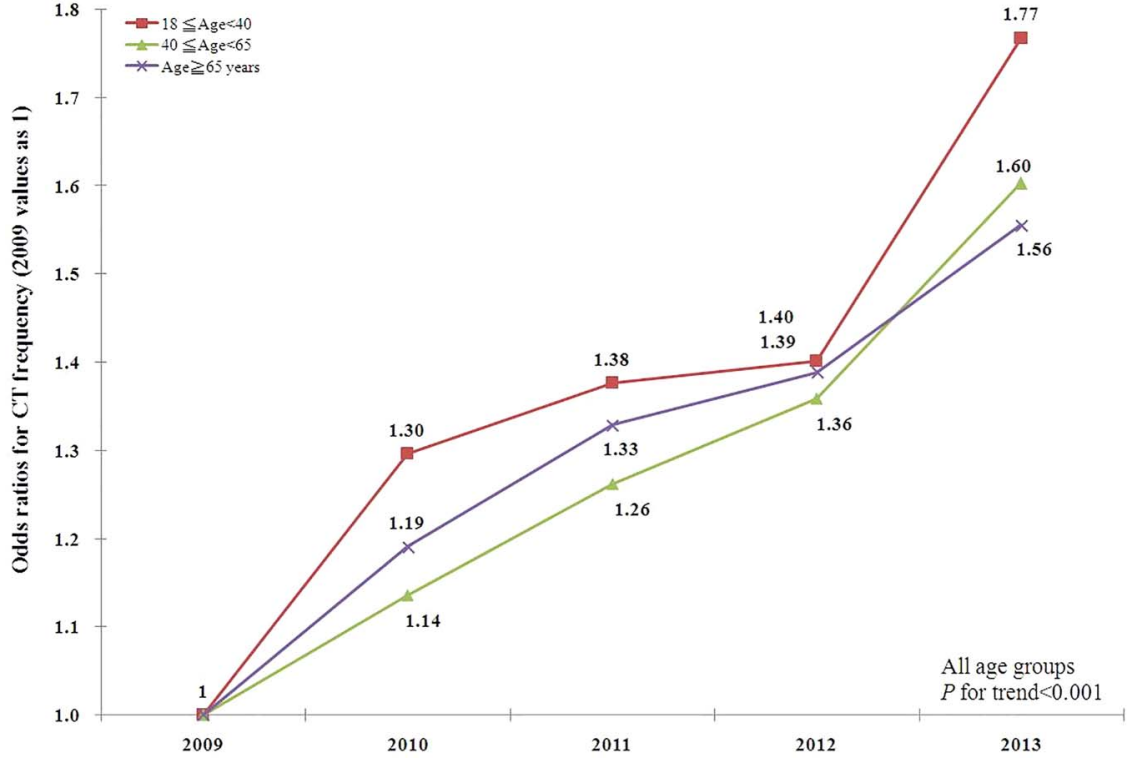

requests from the patients or their families to recheck the patients' condition, since there was no further charge for the repeated CT scan from the National Health Insurance programme. Our hospital would take responsibility for the charge of the repeated CT scan.

In fact, CT scans are not always beneficial to patients. CT scans are associated with significant radiation exposure, compared with other plain radiological diagnostic modalities. The overall median radiation dose of a single CT scan varies from $2 \mathrm{mSv}$ in a routine CT of the head, to $31 \mathrm{mSv}$ in a multiphase CT of the abdomen; the dose varies because of body size and different protocols of the

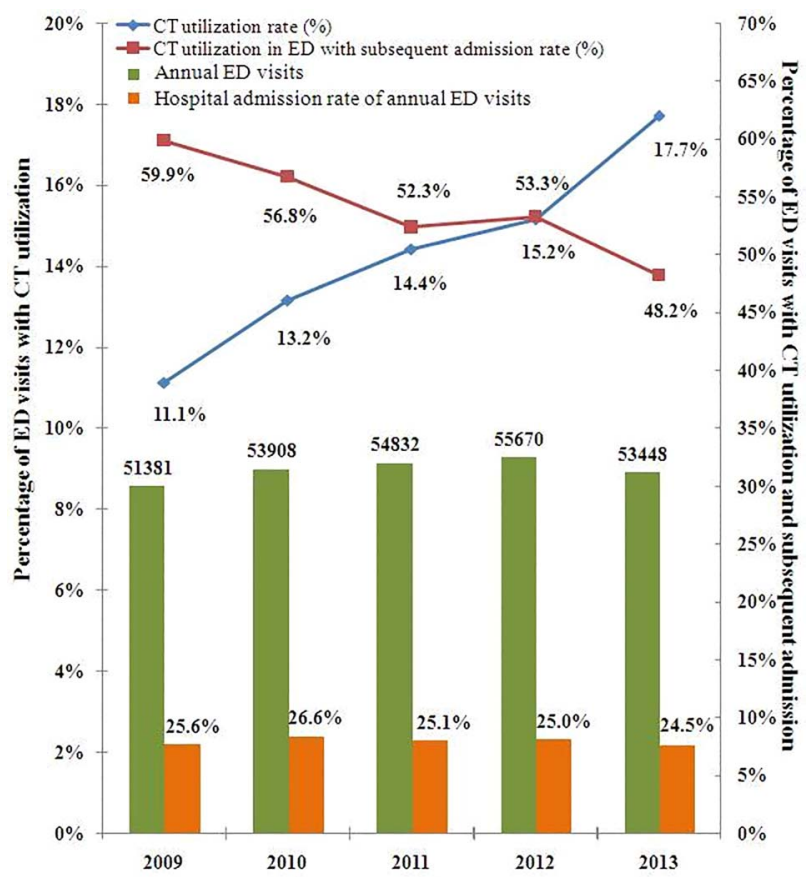

Figure 3 Trends and relationship of annual emergency department (ED) visits and CT utilisation of ED visits with subsequent hospital admission rate during 2009-2013. scans. It is also recognised that there is a radiationrelated cancer risk from CT scans, especially in children. ${ }^{3}{ }^{72-26} \mathrm{CIN}$ is another important issue related to CT scans because CIN is significantly associated with patients' clinical outcomes, including the need for renal replacement, and even subsequent long-term mortality. ${ }^{21}$ Although many strategies have been proposed to prevent the development of CIN, the most effective approach is to decrease the non-clinical factors influencing the physician's decision-making for contrast-enhanced CT utilisation. ${ }^{22-24}$

We provide some suggestions to reduce the non-emergent utilisation of ED CT scans. First, ED CT scans must be utilised in accordance with the established criteria for ED CT after detailed history-taking, with a precise physical examination. Second, it is important to educate patients and explain the risks of CT scans, including allergy to contrast medium, CIN and longterm effects of radiation exposure, such as thyroid disorders and malignancy. Physicians may need training to improve their communication skills. Often, the problem is not the ability of the patient to understand, but the ability of the doctor to communicate effectively and assertively. ED physicians may need additional training to improve their communication skills. Third, senior physicians should audit the orders of CT scans by junior physicians in the ED. ${ }^{1727} 28$

This study had several strengths and limitations. The major strength of this study was the use of a large database from a medical centre in Taiwan, with intrahospital comparisons of patients' data. The costs of ED visits, LOS, and disposition between those with and without CT scans were investigated. The frequency of multiple CT scans during an ED visit was also explored. The major limitations of this study were the retrospective design and confounding factors that could not be excluded. Many of the patients were elderly $(48.03 \%)$ and had multiple symptoms, so the classification of ED visits was challenging. 


\section{CONCLUSION}

This study revealed a steadily increasing rate of ED CT utilisation in a medical centre in Taiwan during 20092013. ED CT utilisation increased at a rate greater than the growth of ED visits. The subsequent admission rate after receiving ED CT did not increase. The reasons for the trend of increasing ED CT utilisation were complex. We speculate that ED CT scans may have been used for rapid screening to facilitate patients' disposition rather than to confirm the diagnosis, under the stress of ED crowding and potential lawsuits. The benefit and harm of ED CT utilisation should be considered according to the physicians' decision-making, which should be based strictly on the patients' clinical presentation. Further investigation is needed to determine whether increasing CT utilisation is efficient and cost-effective.

\section{Author affiliations}

${ }^{1}$ Department of Emergency Medicine, Taichung Veterans General Hospital, Taichung, Taiwan

2Department of Medical Research, Center for Translational Medicine, Taichung Veterans General Hospital, Taichung, Taiwan

${ }^{3}$ School of Medicine, Chung Shan Medical University, Taichung, Taiwan

${ }^{4}$ Institute of Medicine, Chung Shan Medical University, Taichung, Taiwan

${ }^{5}$ School of Medicine, National Yang-Ming University, Taipei, Taiwan

${ }^{6}$ Department of Nursing, College of Health, National Taichung University of Science and Technology, Taichung, Taiwan

${ }^{7}$ Department of Nursing, College of Nursing, Central Taiwan University of Science and Technology, Taichung, Taiwan

${ }^{8}$ Department of Emergency Medicine, Taipei Veterans General Hospital, Taoyuan Branch, Taoyuan, Taiwan

${ }^{9}$ Department of Emergency Medicine, Taipei Veterans General Hospital, Taipei, Taiwan

${ }^{10}$ Institute of Occupational Medicine and Industrial Hygiene, National Taiwan University College of Public Health, Taipei, Taiwan

${ }^{11}$ Department of Radiology, Taichung Veterans General Hospital, Taichung, Taiwan

${ }^{12}$ Department of Medical Research, Taichung Veterans General Hospital, Taichung, Taiwan

${ }^{13}$ Department of Critical Care Medicine, Taichung Veterans General Hospital,

Taichung, Taiwan

${ }^{14}$ Department of Laboratory Medicine, Da-Jia Lee's General Hospital, Lee's Medical Corporation, Taichung, Taiwan

${ }^{15}$ Department of Laboratory Medicine, Yuan-Li Lee's General Hospital, Lee's Medical Corporation, Miaoli, Taiwan

${ }^{16}$ Medical Technology, Jen-Teh Junior College of Medicine, Nursing and Management, Miaoli, Taiwan

${ }^{17}$ Department of Clinical Laboratory, Drug Testing Center, Chung Shan Medical University Hospital, Taichung, Taiwan

Acknowledgements The authors thank the Biostatistics Task Force of Taichung Veterans General Hospital, Taichung, Taiwan, Republic of China, for their assistance and advice in the statistical analysis. They also thank the Clinical Informatics Research and Development Center of Taichung Veterans General Hospital, Taichung, Taiwan, Republic of China, for their assistance in data retrieval from the electronic database and further classification.

Contributors S-YH, M-YL, C-KH and Y-HW developed the study design. T-CL, $\mathrm{C}-\mathrm{YW}, \mathrm{JC}-\mathrm{HT}$ and $\mathrm{C}-\mathrm{YH}$ participated in data acquisition, analyses and interpretation. S-YH and M-SH wrote the manuscript. M-SH and Y-ZC contributed to the critical revision of the manuscript for important intellectual content. All the authors read and approved the final manuscript.

Competing interests None declared.

Ethics approval This study was approved by the Institutional Review Board of Taichung Veterans General Hospital (Number CE13233).
Provenance and peer review Not commissioned; externally peer reviewed.

Data sharing statement No additional data are available.

Open Access This is an Open Access article distributed in accordance with the Creative Commons Attribution Non Commercial (CC BY-NC 4.0) license, which permits others to distribute, remix, adapt, build upon this work noncommercially, and license their derivative works on different terms, provided the original work is properly cited and the use is non-commercial. See: http:// creativecommons.org/licenses/by-nc/4.0/

\section{REFERENCES}

1. Kim SM, Cha RH, Lee JP, et al. Incidence and outcomes of contrast-induced nephropathy after computed tomography in patients with CKD: a quality improvement report. Am J Kidney Dis 2010;55:1018-25

2. Brenner DJ, Hricak $\mathrm{H}$. Radiation exposure from medical imaging: time to regulate? JAMA 2010;304:208-9.

3. Street M, Brady Z, Van Every B, et al. Radiation exposure and the justification of computed tomography scanning in an Australian hospital emergency department. Intern Med $J$ 2009;39:713-9.

4. Solomon RJ, Mehran R, Natarajan MK, et al. Contrast-induced nephropathy and long-term adverse events: cause and effect? Clin J Am Soc Nephrol 2009;4:1162-9.

5. Smith-Bindman R, Lipson J, Marcus R, et al. Radiation dose associated with common computed tomography examinations and the associated lifetime attributable risk of cancer. Arch Intern Med 2009;169:2078-86.

6. Pines JM. Trends in the rates of radiography use and important diagnoses in emergency department patients with abdominal pain. Med Care 2009;47:782-6.

7. Mettler FA Jr, Bhargavan M, Faulkner K, et al. Radiologic and nuclear medicine studies in the United States and worldwide: frequency, radiation dose, and comparison with other radiation sources-1950-2007. Radiology 2009;253:520-31.

8. Prokop M. Radiation dose in computed tomography. Risks and challenges. Radiologe 2008;48:229-42.

9. El-Hajjar M, Bashir I, Khan M, et al. Incidence of contrast-induced nephropathy in patients with chronic renal insufficiency undergoing multidetector computed tomographic angiography treated with preventive measures. Am J Cardiol 2008;102:353-6.

10. Zhou JC, Zheng SW, Yu YX, et al. Trends in computed tomography utilization and association with hospital outcomes in a Chinese emergency department. PLOS ONE 2012;7: e40403.

11. Oh HY, Kim EY, Cho J, et al. Trends of CT use in the adult emergency department in a tertiary academic hospital of Korea during 2001-2010. Korean J Radiol 2013;13:536-40.

12. $\mathrm{Pu} \mathrm{C}, \mathrm{Chou} \mathrm{YJ}$. The impact of continuity of care on emergency room use in a health care system without referral management: an instrumental variable approach. Ann Epidemiol 2016;26:183-8.

13. Huang ST, Wu SC, Hung YN, et al. Effects of continuity of care on emergency department utilization in children with asthma. Am J Manag Care 2016;22:e31-7.

14. Huang MK, Hsu TF, Chiu YH, et al. Risk factors for acute kidney injury in the elderly undergoing contrast-enhanced computed tomography in the emergency department. J Chin Med Assoc 2013;76:271-6.

15. Ho TL, Muo $\mathrm{CH}$, Shen WC, et al. Changing roles of computed tomography in diagnosing acute appendicitis in emergency rooms. QJM 2015;108:625-31.

16. Hess EP, Haas LR, Shah ND, et al. Trends in computed tomography utilization rates: a longitudinal practice-based study. J Patient Saf 2014;10:52-8.

17. Sadegh R, Karimialavijeh E, Shirani F, et al. Head CT scan in Iranian minor head injury patients: evaluating current decision rules. Emerg Radiol 2016;23:9-16.

18. Blok KM, Rinkel GJ, Majoie CB, et al. CT within 6 hours of headache onset to rule out subarachnoid hemorrhage in nonacademic hospitals. Neurology 2015;84:1927-32.

19. Ip IK, Raja AS, Gupta A, et al. Impact of clinical decision support on head computed tomography use in patients with mild traumatic brain injury in the ED. Am J Eemerg Med 2015;33:320-5.

20. Melnick ER, Szlezak CM, Bentley SK, et al. CT overuse for mild traumatic brain injury. Jt Comm J Qual Patient Saf 2012;38:483-9.

21. Tan LA, Kasliwal MK, Traynelis VC. Comparison of CT and MRI findings for cervical spine clearance in obtunded patients without high impact trauma. Clin Neurol Neurosurg 2014;120:23-6. 
22. Tomycz ND, Chew BG, Chang YF, et al. MRI is unnecessary to clear the cervical spine in obtunded/comatose trauma patients: the four-year experience of a level I trauma center. J Trauma 2008:64:1258-63.

23. Brenner DJ, Hall EJ. Computed tomography—an increasing source of radiation exposure. N Engl J Med 2007;357:2277-84.

24. Berrington de Gonzalez A, Mahesh M, Kim KP, et al. Projected cancer risks from computed tomographic scans performed in the United States in 2007. Arch Intern Med 2009;169:2071-7.

25. Lee $\mathrm{Cl}$, Haims $\mathrm{AH}$, Monico EP, et al. Diagnostic CT scans: assessment of patient, physician, and radiologist awareness of radiation dose and possible risks. Radiology 2004;231:393-8.
26. Shiralkar S, Rennie A, Snow M, et al. Doctors' knowledge of radiation exposure: questionnaire study. BMJ 2003;327:371-2.

27. Li CJ, Syue YJ, Tsai TC, et al. The impact of emergency physician seniority on clinical efficiency, emergency department resource use, patient outcomes, and disposition accuracy. Medicine (Baltimore) 2016;95:e2706

28. Hsieh MS, Chiu CS, Chen WC, et al. lodinated contrast medium exposure during computed tomography increase the risk of subsequent development of thyroid disorders in patients without known thyroid disease: a nationwide population-based, propensity score-matched, longitudinal follow-up study. Medicine (Baltimore) 2015;94:e2279. 